\title{
3D Imaging of Dendritic Cells Using Serial Ion-Abrasion Scanning Electron Microscopy (SIA- SEM) Reveals That HIV-1 is Stored Within Surface-connected Membrane Folds.
}

\author{
N. Avishai*, A. Avishai*, M. Hitomi**, H. J. Yu***, A.H. Heuer* and D. McDonald*** \\ *Dept. of Mats. Sci. and Eng., Case Western Reserve University, Cleveland, OH, 44106 \\ **Electron Microscopy Facility, CWRU School of Medicine, Cleveland, OH, 44106 \\ ***Dept. of Molecular Biology \& Microbiology, CWRU School of Medicine, Cleveland, OH, 44106
}

Dendritic cells (DCs) comprise a multivariate family of cell types whose primary function is to initiate immune responses. Myeloid DCs patrol submucosal tissues and engulf pathogens for later degradation and immune presentation to $\mathrm{CD}^{+} \mathrm{T}$ cells. Exposure to bacterial products or inflammatory cytokines at the site of infection results in maturation of the DCs, causing them to migrate to lymphoid tissues where they degrade and present pathogenic peptides to activate antigen specific $\mathrm{CD} 4^{+} \mathrm{T}$ cells. DCs can efficiently process and present HIV; however a portion of the bound virus remains intact and can be transmitted into interacting $\mathrm{CD} 4^{+} \mathrm{T}$ cells through a process known as trans-infection.

Immune activation is a hallmark of acute and chronic HIV infections, ultimately resulting in immune collapse and progression to AIDS. Activation drives myeloid DCs into lymphoid tissues, where the vast majority of HIV replication occurs. DCs can amplify HIV infections within lymphoid tissues by continually picking up and passing on infectious HIV during constitutive immune interactions with $\mathrm{CD}^{+}{ }^{+} \mathrm{T}$ cells. $\mathrm{DCs}$ are therefore potentially important mediators of HIV dissemination in infected individuals, making them an attractive target for new therapies aimed at the prevention and treatment of HIV/AIDS.

Using fluorescent microscopy to follow the intracellular trafficking of virion particles we have shown that DCs trans-infect HIV by forming an "infectious synapse" in which the bound virus is concentrated at the site of contact with T cells. At the same time, the HIV entry receptors CD4, CCR5 and CXCR4 are concentrated on the T cell surface at the synapse, providing an efficient conduit for virus transmission. Activation of the DCs by bacterial products or inflammatory stimuli markedly enhances infectious synapse formation and concomitant trans-infection [1]. We have further shown that HIV is concentrated within a non-endosomal, surface connected "pocket" within activated DCs and that individual virions can traffic from the pocket and enter the $T$ cell at the infectious synapse [2]. Moreover, activated DCs exhibit decreased capacity to process and present HIV peptides to $\mathrm{T}$ cells, suggesting that there is a dynamic balance between trans-infection and antigen processing in DCs. We hypothesize that activation induced down-regulation of endocytosis results in sequestration of infectious HIV in the pocket, where it can be efficiently delivered to CD4 $\mathrm{T}$ cells at the infectious synapse.

To gain insight into the nature of the HIV-containing pocket within DCs, we employed serial ion abrasion scanning electron microscopy (SIA-SEM), using a dual beam FIB. SIA-SEM has recently been employed to visualize the hierarchical organization of molecules and organelles within cells [3]. The technique combines focused ion-beam milling with scanning electron microscopy to generate serial section images that can then be rendered in 3D reconstructions. SIA-SEM has been used extensively to view the internal architecture of semiconductors and other inorganic solids [4]. Application of SIA-SEM for high-resolution 3D imaging of biological specimens has emerged as a powerful new tool for cellular and subcellular imaging in biomedical and clinical research $[5,6]$.

To prepare samples for SIA-SEM, HIV loaded DCs were fixed with $2.5 \%$ glutaraldehyde in $0.1 \mathrm{M}$ 
sodium cacodylate for $1 \mathrm{hr}$., pelleted and fixed an additional hour in glutaraldehyde. Pellets were rinsed with cacodylate buffer and post-fixed with $1 \%$ osmium tetroxide $(\mathrm{OsO} 4)$ in cacodylate for 1 hr. Samples were rinsed and stained with $0.5 \%$ uranyl acetate in $0.1 \mathrm{M}$ acetate buffer for $1 \mathrm{hr}$. The samples were then dehydrated through graded ethyl alcohol (15 min. in $10 \%$ to $100 \% \mathrm{EtOH})$ and propylene oxide (twice for $15 \mathrm{~min}$.). Samples were infiltrated overnight at room temperature with a 1:1 mixture of EMbed 812 resin:propylene oxide, and cured in Embed 812 at $55^{\circ} \mathrm{C}$ for 48 hrs. The blocks were trimmed and surfaced with a microtome on two perpendicular faces to facilitate $\mathrm{Pt}$ deposition and ion abrasion. Blocks were then sputter coated with $30 \mathrm{~nm} \mathrm{Pd}$ and examined in a Nova Nanolab-200 Dual Beam FIB system (FEI). A protective Pt layer was deposited on top of the area of interest. Ion sectioning was performed using a $30 \mathrm{KV}, 1 \mathrm{nA}$ ion beam current to sequentially mill $50 \mu$ sections. SEM imaging was performed using a $2 \mathrm{KV}, 0.84 \mathrm{nA}$ current in immersion lens mode using the through lens detector in backscattered electron mode. Images were acquired in high-resolution format (2048 x1768 pixels) and scanning time of $30 \mu \mathrm{sec}$ per line. Images were viewed, registered, corrected for aspect ratio, and structures were manually or automatically segmented using Image $J$ software. 3D modeling was performed by thresholding cellular surfaces and manually identifying viral particles in each section using Reconstruct software.

Based on fluorescent and thin section TEM imaging, the HIV-containing compartment within DCs had been previously identified as an intracellular, late endosomal vesicle. Our 3D SIA-SEM data clearly demonstrates that the HIV is instead contained within a surface-contiguous region likely to consist of lamellar membrane folds. Our data support our prior observation that the sequestered HIV remains exposed to the extracellular milieu and suggests that the HIV-containing pocket is formed by gathering of HIV bound to membrane ruffles on the cell surface into a single subcellular region within the DC. The application of 3D SIA-SEM to the study of cellular ultrastructure shows great promise to further our understanding of essential features of virus trafficking in and between cells of the immune system.

\section{References}

1. D. McDonald et al., Science (2003) 300: 1295-7.

2. H.J. Yu et al., PLoS Pathogens (2008) 4: e1000134.

3. J.A.W. Heymann, et al., Journal of Structural Biology (2009) 166: 1-7.

4. Yeoh et al., J. Vac. Sci. Technol. B (2007) 25(3): 922-925

5. Knott et al., The Journal of Neuroscience (2008) 28:2959-64.

6. Kidd et al., Micoscopy Today (2010) 18:18-22.

7. Benette et al., PLoS Pathogens (2009) 5:1-10.
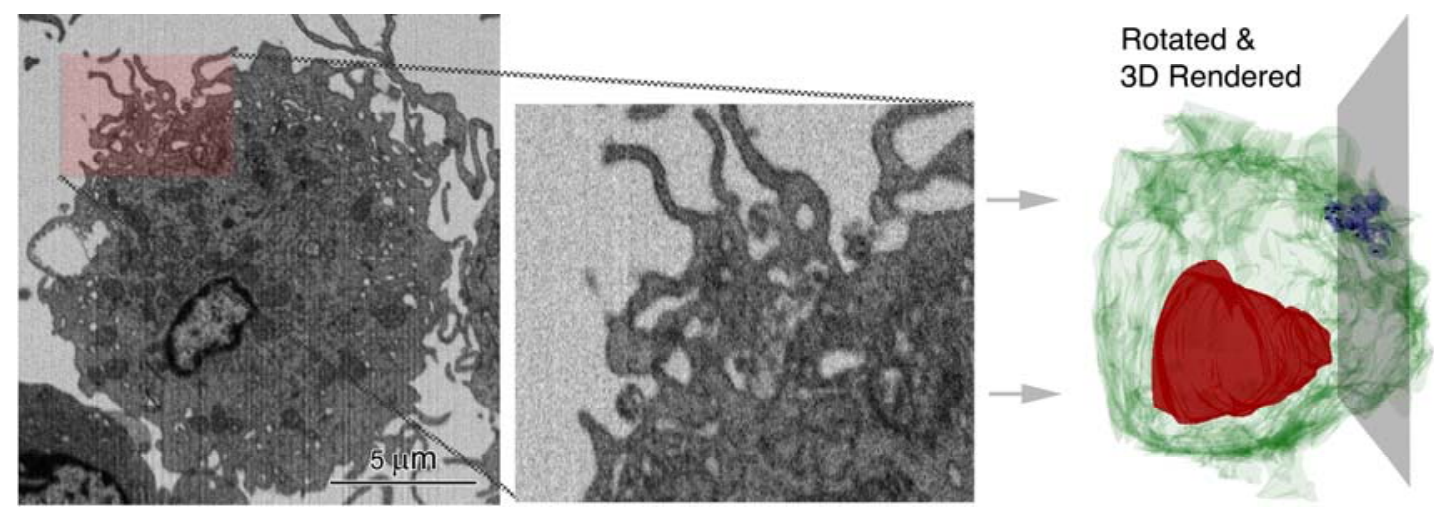

Figure1. 3D image of viral particles in a DC generated using SIA-SEM. a) A SEM image showing a crosssection of an area that was exposed by focused ion beam milling. b) A zoomed in view of the region where viral particles were observed. c) A 3D visualization created from serial sectioning through the DC. 